\title{
Summary of Revisions for the 2013 Clinical Practice Recommendations
}

\section{Revisions to the Standards of Medical Care in Diabetes-2013}

In addition to many small changes related to new evidence since the prior year, and to clarify recommendations, the following sections have undergone more substantive changes:

- Section II.C. Screening for Type 1 Diabetes has been revised to include more specific recommendations.

- Section IV. Prevention/Delay of Type 2 Diabetes has been revised to reflect the importance of screening for and treating other cardiovascular risk factors.

- Section V.C.a. Glucose Monitoring has been revised to highlight the need for patients on intensive insulin regimens to do frequent self-monitoring of blood glucose.

- Section V.D. Pharmacological and Overall Approaches to Treatment has been revised to add a section with more specific recommendations for insulin therapy in type 1 diabetes.

- Section V.F. Diabetes Self-Management Education and Support has been revised to be consistent with the newly revised
National Standards for Diabetes SelfManagement Education and Support.

- Section V.K. Hypoglycemia has been revised to emphasize the need to assess hypoglycemia and cognitive function when indicated.

- Section V.M. Immunization has been updated to include the new Centers for Disease Control and Prevention (CDC) recommendations for hepatitis $\mathrm{B}$ vaccination for people with diabetes.

- Section VI.A.1. Hypertension/Blood Pressure Control has been revised to suggest that the systolic blood pressure goal for many people with diabetes and hypertension should be $<140 \mathrm{mmHg}$, but that lower systolic targets (such as $<130 \mathrm{mmHg}$ ) may be appropriate for certain individuals, such as younger patients, if it can be achieved without undue treatment burden.

- Section VI.A.2. Dyslipidemia/Lipid Management and Table 10 have been revised to emphasize the importance of statin therapy over particular LDL cholesterol goals in high-risk patients.

- Section VI.B. Nephropathy Screening and Treatment and Table 11 have been revised to highlight increased urinary albumin excretion over the terms micro- and macroalbuminuria, other than when discussion of past studies requires the distinction.

- Section VI.C. Retinopathy Screening and Treatment has been revised to include anti-vascular endothelial growth factor therapy for diabetic macular edema.

- Section IX.A. Diabetes Care in the Hospital has been revised to include a recommendation to consider obtaining an $\mathrm{AlC}$ in patients with risk factors for undiagnosed diabetes who exhibit hyperglycemia in the hospital.

\section{Revised Position Statement}

- The position statement "Diagnosis and Classification of Diabetes Mellitus" has been revised slightly to add newer information about monogenic forms of diabetes.

\section{Revisions to the National Standards for Diabetes Self-Management Education and Support}

- The task force report "National Standards for Diabetes Self-Management Education and Support" represents a major revision completed in 2012. 